\title{
Four Blunders in the Electroweak Unification
}

\author{
Gregory L. Light ${ }^{1}$ \\ ${ }^{1}$ Department of Finance, Providence College, 1 Cunningham Square, Providence, Rhode Island 02918, USA \\ Correspondence: Gregory L. Light, Department of Finance, Providence College, 1 Cunningham Square, \\ Providence, Rhode Island 02918, USA. Tel: 1-401-865-2662. E-mail: glight@providence.edu
}

Received: December 29, 2015 Accepted: January 7, 2016 Online Published: January 29, 2016

doi:10.5539/apr.v8n1p188

URL: http://dx.doi.org/10.5539/apr.v8n1p188

\begin{abstract}
We derive the Higgs mass to be $123 \mathrm{GeV}$; we also show that the standard electroweak theory is false by highlighting the fact that the photon nevertheless receives a rest mass along with the $\mathrm{Z}$ boson despite the linear transformation known as the Weinberg angle.
\end{abstract}

Keywords: Weinberg angle, Higgs mass, U(1)xSU(2) false

\section{Introduction}

Based on an established text (Quigg, 2013, in particular, pp. 121-127) we give a critical analysis of the standard electroweak model. We find four logical problems therein: (1) the connection from $L_{\text {gauge }}$ to $L_{\text {leptons }}$, (2) failure to deduce the model implied Higgs mass to be $123 \mathrm{GeV}$, (3) failure of the linear transformation of the Weinberg angle to remove the photon as a recipient of a rest mass, and (4) unit incongruence in the identification of $e=g \sin \theta_{w}$.

An extensive literature research has shown no similar analyses to ours in existence. Weinberg after his paper in 1967 established a lower bound of the Higgs mass in 1976; Kant (2012) employed minimal supersymmetric extension of the Standard Model (MSSM) to a numerical asymptotic expansion of the Higgs mass; Capdevilla et al (2015) sought to build a model to recover the well-publicized Higgs mass of $125 \mathrm{GeV}$. In theoretical modeling, Bhattacharjee and Majumdar (2011) pursued an electroweak unification without spontaneous symmetry breaking. On the whole, researchers have taken the electroweak theory for granted and sought only to lay out the evolutionary details of how electromagnetism and the weak nuclear force became separated (see, e.g., Bhattacharyya, 2011).

We present our findings in the next Section 2 and conclude in Section 3.

\section{Deconstructing U(1) $\times \mathbf{S U}(2)$}

The Standard Electroweak Model is built upon a Lagrangian as assembled from the origin of the forces ("gauge"), their interactions with (anti) electrons and (anti) neutrinos ("leptons"), and the source of rest masses ("scalar"). The notation below follows from Quigg.

\subsection{The Extraction of $\boldsymbol{A}$ from Lgauge is Invalid}

One has:

$$
L_{\text {gauge }}=-\frac{1}{4} F_{\mu \nu}^{l} F^{l \mu v}-\frac{1}{4} f_{\mu \nu} f^{\mu \nu},
$$

where in particular,

$$
-\frac{1}{4} f_{\mu \nu} f^{\mu \nu}=\frac{1}{2} \varepsilon_{0}\left(\|E\|^{2}-\|c B\|^{2}\right)\left(J / m^{3}\right),
$$

an electromagnetic wave, which has no electric charge. However,

$$
\begin{aligned}
& f_{\mu \nu}=\partial_{v} A_{\mu}-\partial_{\mu} A_{v} \\
& \text { has } A_{t}=\frac{e}{4 \pi \varepsilon_{0} r}(\text { volt }) ;
\end{aligned}
$$

i.e., $A_{t}$ is not a photon, yet the literature treats 


$$
\left\{A, b^{(1)}, b^{(2)}, b^{(3)}\right\}
$$

as four bosonic fields, with $A$ serving as the photon. That $A$ carrying an electric charge has nevertheless been equated to a photon is due to the false assertion that

$$
f_{\mu v} f^{\mu v}
$$

implies Maxwell Equations so that the photon is responsible for electromagnetism (resulting in a confused state of the logic between electromagnetism and electromagnetic waves, see, e.g., Pai, 2015, and cf. Light, 2015). This century-old assertion is false because in order to derive the laws of Ampere's and Faraday's by applying the Hodge (1903-1975) star operator (as developed from the algebra by Grassmann, 1809-1877, and the calculus by Cartan, 1869-1951) to

$$
f_{\mu v} f^{\mu v} \equiv \varepsilon_{o} c^{2} f_{\mu \nu} f^{\mu v},
$$

one would have to factor the above product asymmetrically, as in

$$
-c^{-2}\|E\|^{2}=\left(0, E_{x}, E_{y}, E_{z}\right) \cdot\left(0,-E_{x} / c^{2},-E_{y} / c^{2},-E_{z} / c^{2}\right) .
$$

Such a factorization is analogous to factoring a square as a rectangle, with side ratio $c^{2}: 1$, effectively forcing electromagnetism out of electromagnetic waves, which is invalid since $B$ is a sideways force in the former but is symmetric with $E$ in the latter.

\subsection{Failure to Obtain the Implied Higgs Mass}

One has:

$$
\begin{aligned}
& L_{\text {scalar }}=\left(D^{\mu} \phi\right)^{\dagger}\left(D_{\mu} \phi\right)-V\left(\phi^{\dagger} \phi\right), \\
& D_{\mu}:=\partial_{\mu}+\frac{i g^{\prime}}{2} A_{\mu} Y+\frac{i g}{2} \tau \bullet b_{\mu} ; \\
& V\left(\phi^{\dagger} \phi\right)=\mu^{2}\left(\phi^{\dagger} \phi\right)+|\lambda|\left(\phi^{\dagger} \phi\right)^{2}
\end{aligned}
$$

has units:

$$
\frac{J^{2}}{m^{6}}=(J s)^{2} \cdot\left(\frac{1 / s^{2}}{m^{6}}\right)+(J s)^{2}\left(m^{3} s\right)^{2} \cdot\left(\frac{1 / s^{4}}{m^{12}}\right)
$$

so that

$$
\phi(t, x, y, z)=\omega(t, x, y, z) / m^{3}
$$

is the angular frequency density of the wave associated with a Higgs boson.

$$
\begin{aligned}
& \text { At } \phi=\left(\sqrt{\frac{-\mu^{2}}{|\lambda|}}\right), V=0 ; \\
& \text { set } \omega_{0}:=\sqrt{\frac{-\mu^{2}}{|\lambda|}} m^{3} ; \text { then } \frac{\omega_{0}}{\sqrt{2}} \text { minimizes } V . \\
& \text { Define } \frac{\eta}{\sqrt{2}}:=\omega-\frac{\omega_{0}}{\sqrt{2}} ; \\
& \text { then } m^{6} V=\mu^{2} \omega^{2}+|\lambda| \omega^{4} \equiv \mu^{2}\left(\frac{\eta}{\sqrt{2}}+\frac{\omega_{0}}{\sqrt{2}}\right)^{2}+|\lambda|\left(\frac{\eta}{\sqrt{2}}+\frac{\omega_{0}}{\sqrt{2}}\right)^{4} \\
& =\left(\frac{\eta}{\sqrt{2}}\right)^{2}\left(\mu^{2}+6|\lambda| \cdot \frac{-\mu^{2}}{2|\lambda|}\right)+(\text { the other terms *) } \\
& =-\mu^{2} \eta^{2}+(*) .
\end{aligned}
$$


Substituting $\left(\frac{\eta}{\sqrt{2}}\right)$ into (1.3),

we have

$$
\begin{aligned}
& \left(i \hbar \frac{\partial}{\partial x^{\mu}} \frac{\eta}{\sqrt{2}} / m^{3}\right)^{\dagger}\left(i \hbar \frac{\partial}{\partial x_{\mu}} \frac{\eta}{\sqrt{2}} / m^{3}\right)-V\left(\frac{\eta}{\sqrt{2}}\right) \\
& =\frac{1}{2} m^{-6}\left[\left(\hbar \frac{\partial}{\partial x^{\mu}} \eta\right)^{\dagger}\left(\hbar \frac{\partial}{\partial x_{\mu}} \eta\right)+2 \mu^{2} \eta^{2}\right]-\left(^{*}\right) \\
& =\frac{1}{2} m^{-6}\left[\left(E_{\text {Higgs }}-p_{\text {Higgs }} c\right)^{2}-\left(m_{0} c^{2}\right)^{2}\right]-\left(^{*}\right) . \\
& \text { Then }\left[\min L_{\text {scalar }}\right] \Rightarrow\left[m_{0, \text { Higgs }} c^{2}=\sqrt{-2 \mu^{2}} \cdot \frac{\omega_{0}}{\sqrt{2}}=\hbar \cdot \frac{\omega_{0}}{\sqrt{2}}\right] .
\end{aligned}
$$

Now,

substituting $b_{t}^{(1)}, b_{t}^{(2)}$ and $\frac{\omega}{\sqrt{2}}$ into (1.3), we have:

$$
\begin{aligned}
& \left(\frac{i g \hbar}{2} \tau \cdot b_{t} \cdot \frac{\omega}{\sqrt{2}}\right)^{\dagger}\left(\frac{i g \hbar}{2} \tau \cdot b_{t} \cdot \frac{\omega}{\sqrt{2}}\right) \\
& =\frac{1}{4} g^{2} \hbar^{2}\left(\frac{\omega}{\sqrt{2}}\right)^{2}\left(\begin{array}{cc}
0 & b_{t}^{(1)}+b_{t}^{(2)} i \\
b_{t}^{(1)}-b_{t}^{(2)} i & 0
\end{array}\right) \cdot\left(\begin{array}{cc}
0 & b_{t}^{(1)}-b_{t}^{(2)} i \\
b_{t}^{(1)}+b_{t}^{(2)} i & 0
\end{array}\right) \\
& =\frac{1}{2} g^{2} \hbar^{2}\left(\frac{\omega}{\sqrt{2}}\right)^{2}\left(\left|\frac{b_{t}^{(1)}+b_{t}^{(2)} i}{\sqrt{2}}\right|^{2}+\left|\frac{b_{t}^{(1)}-b_{t}^{(2)} i}{\sqrt{2}}\right|^{2}\right) \\
& \equiv \frac{1}{2} g^{2} \hbar^{2}\left(\frac{\omega}{\sqrt{2}}\right)^{2}\left(\left|W_{t}^{-}\right|^{2}+\left|W_{t}^{+}\right|^{2}\right)=g^{2} \hbar^{2}\left(\frac{\omega}{\sqrt{2}}\right)^{2}\left|W_{t}^{ \pm}\right|^{2},
\end{aligned}
$$

implying that $m_{0, W^{ \pm}} c^{2}=g \hbar \frac{\omega}{\sqrt{2}}$.

Substituting $W^{ \pm}$into the charged part in

$$
\begin{aligned}
& L_{\text {leptons (Left) }}=\bar{L} i \gamma^{\mu}\left(\partial_{\mu}+\frac{i g^{\prime}}{2} A_{\mu} Y+\frac{i g}{2} \tau \cdot b_{\mu}\right) L \text {, we have: } \\
& i \gamma^{\mu} \cdot \frac{i g}{2} \bar{L}\left(\begin{array}{cc}
0 & b^{(1)}{ }_{\mu}-b^{(2)}{ }_{\mu} i \\
b_{\mu}^{(1)}+b_{\mu}^{(2)} i & 0
\end{array}\right) L \\
& =-\frac{\sqrt{2}}{2} g \gamma^{\mu} \bar{L}\left(\begin{array}{cc}
0 & W^{+} \\
W^{-} & 0
\end{array}\right) L \\
& =-\frac{\sqrt{2}}{2} g \gamma^{\mu}\left(\begin{array}{ll}
\bar{v}_{L} & \bar{e}_{L}
\end{array}\right)\left(\begin{array}{cc}
0 & W^{+} \\
W_{\mu}^{-} & 0
\end{array}\right)\left(\begin{array}{l}
v_{L} \\
e_{L}
\end{array}\right) \\
& =-\frac{\sqrt{2}}{2} g \gamma^{\mu}\left(\bar{v}_{L} W_{\mu}^{+} e_{L}+\bar{e}_{L} W_{\mu}^{-} \nu_{L}\right) \\
& =-\frac{\sqrt{2}}{4} g\left(\bar{v} \gamma^{\mu}\left(\begin{array}{cc}
O_{2} & O_{2} \\
O_{2} & 2 I_{2}
\end{array}\right) e W_{\mu}^{+}+\bar{e} \gamma^{\mu}\left(\begin{array}{cc}
O_{2} & O_{2} \\
O_{2} & 2 I_{2}
\end{array}\right) \nu W_{\mu}^{-}\right) \\
& =-\sqrt{\frac{G_{F} \cdot\left(m_{0, W^{ \pm}} c^{2}\right)^{2}}{\sqrt{2}}} \cdot\left(\bar{v} \gamma^{\mu}\left(\begin{array}{cc}
O_{2} & O_{2} \\
O_{2} & 2 I_{2}
\end{array}\right) e W_{\mu}^{+}+\bar{e} \gamma^{\mu}\left(\begin{array}{cc}
O_{2} & O_{2} \\
O_{2} & 2 I_{2}
\end{array}\right) \nu W_{\mu}^{-}\right)
\end{aligned}
$$

(by low-energy interaction, [Quigg], p. 110;

$G_{F} \equiv$ the Fermi constant $\left.\approx 1.17 \times 10^{-5}(\mathrm{GeV})^{-2}\right)$. 


$$
\begin{aligned}
& \text { Thus, } \frac{g^{2}}{8}=\frac{G_{F} \cdot\left(m_{0, W^{ \pm}} c^{2}\right)^{2}}{\sqrt{2}}, \\
& \text { but } m_{0, W^{ \pm}} c^{2}=g \hbar \frac{\omega_{0}}{\sqrt{2}}(\text { from }(1.7)), \\
& \text { so } \hbar \frac{\omega_{0}}{\sqrt{2}}=\left[\frac{\sqrt{2}}{8} \times 1.17^{-1} \times 10^{5}(\mathrm{GeV})^{2}\right]^{\frac{1}{2}} \\
& =\left(0.177 \times 0.85 \times 10^{5}\right)^{\frac{1}{2}} \mathrm{GeV} \\
& =\sqrt{0.151 \times 10^{5}} \mathrm{GeV}=\sqrt{15109} \mathrm{GeV} \\
& =122.92 \mathrm{GeV}=m_{0, \text { Higgs }} c^{2} .
\end{aligned}
$$

2.3 Higgs Field Makes Photons have Rest Masses, too.

$$
\begin{aligned}
& \operatorname{Set}\left(\begin{array}{l}
Z_{0, t} \\
A_{0, t}
\end{array}\right)=\frac{1}{\sqrt{2}} \frac{1}{\sqrt{g^{\prime 2}+g^{2}}}\left(\begin{array}{cc}
g & -g^{\prime} \\
g^{\prime} & g
\end{array}\right)\left(\begin{array}{c}
b^{(3)}{ }_{t} \\
A_{t}
\end{array}\right), \text { or } \\
& \left(\begin{array}{c}
b^{(3)} \\
A_{t}
\end{array}\right)=\frac{\sqrt{2}}{\sqrt{g^{\prime 2}+g^{2}}}\left(\begin{array}{cc}
g & g^{\prime} \\
-g^{\prime} & g
\end{array}\right)\left(\begin{array}{c}
Z_{0, t} \\
A_{0, t}
\end{array}\right),
\end{aligned}
$$

(where the factor $\sqrt{2}$ is needed in anticipation of shifting the energy from $\mathbf{A}$ to $\mathrm{b}^{(3)}$, to make the electrically charged A transformed away, a calculation detail that has been overlooked in the literature, see Quigg). By the preceding (1.10) the literature then sought to prevent the same $\phi$ that has assigned rest masses to $W^{ \pm}$from similarly assigning a rest mass to the photon $A_{0}$ :

$$
\begin{aligned}
& \left\{\left[\frac{i g^{\prime} \hbar}{2} A_{t}\left(\begin{array}{cc}
-1 & 0 \\
0 & -1
\end{array}\right)+\frac{i g \hbar}{2} b^{(3)}\left(\begin{array}{cc}
1 & 0 \\
0 & -1
\end{array}\right)\right]\left(\begin{array}{c}
0 \\
\phi^{0}
\end{array}\right)\right\}^{\dagger} \\
& \cdot\left\{\left[\frac{i g^{\prime} \hbar}{2} A_{t}\left(\begin{array}{cc}
-1 & 0 \\
0 & -1
\end{array}\right)+\frac{i g \hbar}{2} b^{(3)}{ }_{t}\left(\begin{array}{cc}
1 & 0 \\
0 & -1
\end{array}\right)\right]\left(\begin{array}{c}
0 \\
\phi^{0}
\end{array}\right)\right\} \\
& =\frac{1}{4}\left(\hbar \frac{\omega_{0}}{\sqrt{2}}\right)^{2}\left(g^{\prime} A_{t}-g b^{(3)}{ }_{t}\right)^{2} \text {, }
\end{aligned}
$$

which corresponded to the crucial term in Equation (7) of Weinberg (1967)

that would lead to massless photons.

Substituting (1.10) into the square-root of the above, the literature appeared to have succeeded in its intent:

$$
\begin{aligned}
& \frac{1}{2}\left(\hbar \frac{\omega_{0}}{\sqrt{2}}\right) \cdot \frac{\sqrt{2}}{\sqrt{g^{\prime 2}+g^{2}}}\left[g^{\prime}\left(-g^{\prime} Z_{0, t}+g A_{0, t}\right)-g\left(g Z_{0, t}+g^{\prime} A_{0, t}\right)\right] \\
& =-\frac{\sqrt{2}}{2}\left(\hbar \frac{\omega_{0}}{\sqrt{2}}\right) \sqrt{g^{\prime 2}+g^{2}} Z_{0, t}, \text { so that } \\
& m_{0, Z_{0}} c^{2}=\frac{\sqrt{2}}{2} \sqrt{g^{\prime 2}+g^{2}}\left(\hbar \frac{\omega_{0}}{\sqrt{2}}\right) \text { and } \\
& m_{0, A_{0}} c^{2}=0, \text { as desired. }
\end{aligned}
$$

However, the matrix multiplication in (1.11)

$$
\left(\begin{array}{cc}
1 & 0 \\
0 & -1
\end{array}\right)\left(\begin{array}{c}
0 \\
\phi^{0}
\end{array}\right)
$$


is invalid. To elaborate: (i) $\left(\begin{array}{c}0 \\ \phi^{0}\end{array}\right) \neq 0 \cdot e_{x}+\phi^{0} \cdot e_{z}$ for $\sigma_{z}:=\left(\begin{array}{cc}1 & 0 \\ 0 & -1\end{array}\right)$ to operate on, i.e., the initially postulated $\phi \equiv\left(\begin{array}{c}\phi^{+} \\ \phi^{0}\end{array}\right)$ does not have a Cartesian directional content since $\phi$ can also be represented as $\left(\begin{array}{l}\phi^{0} \\ \phi^{+}\end{array}\right)$. On the other hand, $\sigma_{z}$ describes the wavefunction of an electron situated in a uniform magnetic field $B=e_{z}$ tesla, resulting in an electric field momentum direction $e_{x}$ (representing the state "up") or - $e_{z}$ (representing the state "down," which results from a 3-dimensional rotation from $e_{x}$, cf. Light, 2013). As such, a linear combination of $e_{x}$ and $-e_{z}$ by two multiples that are devoid of any directional intent is meaningless, to be sure,

$$
\left(\begin{array}{cc}
1 & 0 \\
0 & -1
\end{array}\right)\left(\begin{array}{c}
0 \\
\phi^{0}
\end{array}\right) \neq\left(\begin{array}{cc}
1 & 0 \\
0 & -1
\end{array}\right)\left(\begin{array}{c}
\phi^{0} \\
0
\end{array}\right) \text {. }
$$

(ii) $\sigma_{z} \neq$ the linear mapping $\left[\left(\begin{array}{l}1 \\ 0\end{array}\right) \mapsto 1,\left(\begin{array}{l}0 \\ 1\end{array}\right) \mapsto i\right]$ (for then $\sigma_{z}^{2}=I \equiv 1+i$ would imply $\sigma_{z}^{4}=I \equiv(1+i)^{2} \neq 1+i \equiv I$, a contradiction ) so that $\phi \equiv \phi^{+}+\phi^{0} i$ as a "complex doublet" does not lend to the calculation $\left(\begin{array}{cc}1 & 0 \\ 0 & -1\end{array}\right)\left(\begin{array}{c}0 \\ \phi^{0}\end{array}\right)$, either.

(iii) In the inner product of (1.11), $\sigma_{z}$ serves as a vector in $R^{4}$ and is thus not an operator; as such, $\phi^{0}$ must enter (1.11) as a scalar. In fact, the label of the Lagrangian is scalar, but if the literature had substituted $\phi^{0}$ into (1.11) (as done in the above (1.7)) instead of $\left(\begin{array}{c}0 \\ \phi^{0}\end{array}\right)$, then the result would have been

$$
\begin{aligned}
& \left\{\left[\frac{i g^{\prime} \hbar}{2} A_{t}\left(\begin{array}{cc}
-1 & 0 \\
0 & -1
\end{array}\right)+\frac{i g \hbar}{2} b^{(3)}\left(\begin{array}{cc}
1 & 0 \\
0 & -1
\end{array}\right)\right] \phi^{0}\right\}^{\dagger} \\
& \cdot\left\{\left[\frac{i g^{\prime} \hbar}{2} A_{t}\left(\begin{array}{cc}
-1 & 0 \\
0 & -1
\end{array}\right)+\frac{i g \hbar}{2} b^{(3)} t_{t}\left(\begin{array}{cc}
1 & 0 \\
0 & -1
\end{array}\right)\right] \phi^{0}\right\} \\
& =\frac{1}{4}\left(\hbar \frac{\omega_{0}}{\sqrt{2}}\right)^{2}\left[\left(g^{\prime} A_{t}+g b_{t}^{(3)}\right)^{2}+\left(g^{\prime} A_{t}-g b_{t}^{(3)}\right)^{2}\right],
\end{aligned}
$$

with an additional term $\left(g^{\prime} A_{t}+g b^{(3)}{ }_{t}\right)$ (cf. Equations (1.11), (1.12)); i.e., the linear transformation as characterized by the Weinberg angle $\theta_{w}=\arccos \left(\frac{g}{\sqrt{g^{\prime 2}+g^{2}}}\right)$ would have been found to fail to prevent $A_{0, t}$ from coexisting with $Z_{0, t}$ to receive a rest mass from $\phi^{0}$ so that $m_{0, A_{0}} c^{2}>0$.

2.4 The Identification of $g \sin \theta_{w}=e$ is Invalid
After replacing $A_{\mu}$ and $b^{(3)}{ }_{\mu}$ with $A_{0, \mu}$ and $Z_{0, \mu}$ in $L_{\text {leptons }(L)}, \quad$ the $e_{g g}$ literature arrived at a term $\frac{g g^{\prime}}{\sqrt{g^{2}+g^{\prime 2}}} \bar{e} \gamma^{\mu} e A_{\mu}$ ( Quigg, p. 126, Equation (6.3.47)) and proceeded to identify $\frac{g g}{\sqrt{g^{2}+g^{\prime 2}}}=e$, (Weinberg, 1967, Equations (14), (15)) but this in invalid since the " $e$ " originating from the wavefunctions in (1.8) carries a unit of $\left(1 / \sqrt{m^{3}}\right)$ so that $\bar{L} L$ yields a probability density, with $g$ or $\mathrm{g}^{\prime}$ being a unit-free

positive real number. In fact,

$$
\begin{aligned}
& g^{\prime}=\alpha \equiv \frac{e_{A} e_{L}}{4 \pi \varepsilon_{0} \hbar c}=\frac{\frac{e_{A} e_{L}}{4 \pi \varepsilon_{0} \lambda}}{h v / 2 \pi}=\frac{A e_{L}}{h v / 2 \pi}, \text { so that } \\
& g^{\prime} \cdot(h v / 2 \pi)=A e_{L},
\end{aligned}
$$

meaning that if we express the classical electric potential energy between $e_{A}$ and $e_{L}$ as $h v / 2 \pi$, then $g^{\prime} \cdot(h v / 2 \pi)$ recovers the same result. Note that $\lambda$ has unit $(\mathrm{m} / \mathrm{cycle})$, yet $E=\hbar \omega \equiv h v$ has a unit of $(J \cdot \mathrm{rad})$, so that $h v / 2 \pi$ is required in the denominators of the above expressions. For neater exposition, we could also identify $g^{\prime}=\alpha / 2 \pi$. 


\section{Conclusion}

In this paper, we have shown that $A_{0}$ in SU(2) would still possess a rest mass; since photons do not have rest masses, photons are not the claimed gauge bosons of electromagnetism and hence the Standard Model fails to explain the electromagnetic force.

Otherwise, the identity

$$
\alpha \equiv \frac{\frac{e e}{4 \pi \varepsilon_{0} \lambda}}{h v / 2 \pi}
$$

suggests that whatever the distance $\lambda$ separating any two electrons may be, there is the universal probability of

$$
\alpha \approx \frac{1}{137}
$$

that there exists an electromagnetic wave of wave length

$$
\lambda \equiv c / v
$$

to connect the two electrons, implying an instant communication between the two electrons and supporting our proposed combined spacetime 4-manifold (cf. e.g., [Light, 2015]), where the wave universe has a quotient-space topology so that

$$
0 \equiv \lambda \text { in }[0, \lambda]
$$

\section{References}

Bhattacharjee, S., \& Majumdar, P. (2011). Gauge-free electroweak theory: Radiative effects. Physical Review D, 83, 085019 (7 pages). http://dx.doi.org/10.1103/PhysRevD.83.085019

Bhattacharyya, G. (2011). A pedagogical review of electroweak symmetry breaking scenarios. Reports on Progress in Physics, 74, 26201-26238. http://dx.doi.org/10.1088/0034-4885/74/2/026201

Capdevilla, R. M., Delgado, A., \& Martin, A. (2015). Light stops in a minimal U(1)X extension of the MSSM. Physical Review D, 92, 115020 (8 pages). http://dx.doi.org/10.1103/PhysRevD.92.115020

Kant, P. (2012). Three-loop calculation of the higgs boson mass in supersymmetry. Journal of Physics: Conference Series, 368, 12053-12059. http://dx.doi.org/10.1088/1742-6596/368/1/012053

Light, G. L. (2015). Remarks on the standard model. International Journal of Applied Mathematics, 28, 651-666. http://diogenes.bg/ijam/contents/2015-28-6/2/2.pdf

Light, G. L. (2013). The spinning motions of all fermions and bosons as implied by Pauli matrices containing complex conjugates in a combined spacetime 4-manifold. Applied Physics Research, 5, 37-45. http://dx.doi.org/10.5539/apr.v5n4p37

Pai, S. T. (2015). Can the photon be both massive and massless? Physics Essays 28, 55-62. http://dx.doi.org/10. 4006/0836-1398-28.1.55

Quigg, C. (2013). Gauge Theories of the Strong, Weak, and Electromagnetic Interactions. Princeton: Princeton University Press.

Weinberg, S. (1976). Mass of the Higgs boson. Physical Review Letters, 36, 294-296. http://dx.doi.org/10.1103/ PhysRevLett.36.294

Weinberg, S. (1967). A model of leptons, Physical Review Letters, 19, 1264-1266. http://dx.doi.org/10.1103/Phys RevLett.19.1264

\section{Copyrights}

Copyright for this article is retained by the author(s), with first publication rights granted to the journal.

This is an open-access article distributed under the terms and conditions of the Creative Commons Attribution license (http://creativecommons.org/licenses/by/3.0/). 